\title{
Updating the descriptive biopsychosocial approach to fit into a formal person-centered dynamic coherence model
}

\author{
Thomas Fröhlich MD PhD Dipl. Biol ${ }^{a}$, F.F. Bevier Dipl. Phys ${ }^{b}$, Alicja Babakhani MD ${ }^{c}$, Hannah \\ H. Chisholm BHSc (Hons) ${ }^{d}$, Peter Henningsen MD ${ }^{\mathrm{e}}$, David S. Miall PhD', Seija Sandberg PhD \\ FRCPsych (Lond) ${ }^{g}$ and Arbogast Schmitt PhD ${ }^{\mathrm{h}}$
}

\author{
a Paediatrician, Allergist, Psychotherapist, Pediatric and Family Medicine Private Practice, Bammental, \& Vice President \\ (Western Europe), European Society for Person Centered Healthcare, Heidelberg, Germany \\ b Physicist, Bussole InformationsVerlag, Winden, Germany \\ c GP and Family Medicine Private Practice, Reichartshausen, Germany \\ d Research Assistant, Heidelberg Metasystems $\mathrm{GmbH}$, Heidelberg, Germany \\ e Professor, Chair of Psychosomatic Medicine, Klinikum Rechts der Isar, Munich Technical University, Psychosomatic \\ Medicine, Munich, Germany \\ f Professor Emeritus, English and Film Studies Department, University of Alberta, Edmonton, AB, Canada \\ g Consultant Child and Adolescent Psychiatrist, Eira Hospital, Helsinki, Finland \\ h Professor Emeritus, Seminar für Klassische Philologie, Philipps-University Marburg, Marburg \& Honorary Professor, Freie \\ Universität, Berlin, Germany
}

\section{Keywords}

Biology, biopsychosocial model, Cartesian duality, dynamic coherence provider, epistemology, heuristics, informatics, person-centered healthcare, physics, ontology, salutogenesis, stochastic changes

\section{Correspondence address}

Dr. Thomas Fröhlich, In den Brunnerwiesen 4, 69245 Bammental, Germany. E-mail: drfroehlich@me.com

Accepted for publication: 3 July 2016

\section{Introduction}

A 'biopsychosocial' approach to healthcare, as initially described by George Engel in his celebrated paper published within the journal Science [1], has been in common use for many decades. Contained in the term 'biopsychosocial' is the assumption of an internal homogeneity between today's different conceptual approaches to understanding individual persons as a unit and as complex dynamic wholes. In his initial publication, Engel outlined a preliminary, hypothetical framework and stated that a corresponding clinical model was needed. This still has not been achieved and currently this preliminary framework is erroneously understood as a model. Such a model requires the establishment of a basis for both science and humanities, a formidable task.

In order to establish such a basis we used two methods in parallel. First, we introduced a formal logical notation that allows us to address those features of time that necessarily have to be taken into account in humanities, but are not extensively dealt with in sciences such as informatics, physics and biology. Second, with regard to the qualifying and specifying of the aspects of time, we referred back to human thinking at a time when science and humanities were not divided into two seemingly autonomous fields of study. We introduced the phrase 'dynamic coherence provider' as a suggested contemporary term for being given or hypostasised as an underlying potential. Except for the indeterminableness of future, there is nothing mysterious about such a dynamic coherence provider: a motorbike is also one - as long as its screws hold tight so that proceeding occurrence (with its indeterminacy) can be expected.

Then, on a non-stochastic level, we can differentiate between changes of states and something underlying that provides and ensures the internal coherence within these changes. One of the results of the underlying, provided coherence is that changes do not happen just once, but instead have the potential to be re-iterated. This in turn provides sequential coherence; that is a maintenance of an identity over a given period of observed time. Therefore, the way we can apply a formal concept of natural identity, that, while being compatible with scientific formalisation, also allows for topics that are commonly subsumed exclusively under philosophy, such as the uniqueness of a person, to be addressed.

We are clearly opposed to Cartesian concepts, instead applying a potential-with-reality differentiation extensively studied in Aristotelian and scholastic philosophy. Its heuristic makes a clear distinction: whereas states and changes of states can be observed empirically by direct measurement, that which is said about a future and hence only a potential development, necessarily is and has the 
form of, a hypothesis. Thus, the level of states and changes of states that have occurred and the level of changes of states that may, or may not, occur, must be dealt with differently. What is achieved by this differentiation is a more realistic, truly empirical, concept of time. Whereas such a distinction is in common use in research in a more implicit way, it plays a pivotal role in our approach. Here, different from the common approach, the underlying concept of time consists of a non-continuous, time and position-bound, specific and specifying character of time. As seen from 'outside', this type of time is encapsulated within the examined objects and as seen and lived from inside out, the latent, qualitative, temporal potential is, or may be, unfolded via its realisation manifestation.

In a balance of the two foci, the level of already realised items and those that are only expected to happen, the approach allows spatiotemporal individuality to be better addressed. In applying the latter as a concept, we may understand a person as "an integrated, purposeful whole individual, rather than a disorganized set of reactive impulses" [2]. Whereas in the common objectifying approach to time a spatial metaphor is usually a good enough approximation, with time mapped as a fourth, seemingly continuous dimension in an approach that also takes subjectivity into account, then both the uniqueness of every moment and the uniqueness of every moment's 'inside' (in qualitative spatial and qualitative procedural, temporal terms) must be recognised. The present, unique moment, together with its still given potentiality concerning its next future, differentiates and qualifies a corresponding inside as being special and as not being the same as something on its outside.

In also addressing what makes each individual a unique coherence and not only a bodily and mental coherence as given in objective, hence general terms, we conceptualise her or him also as the source of processes that start in her or him and that, via this fixed and maintained initiating position, have a unique, initially irreversible direction and a corresponding directedness towards the world outside and inside.

For this not only descriptive, but also formal combination of the two different approaches, the one addressing objective aspects and the other subjective ones, we introduce a symbolisation for the aforementioned discreteness of inside-to-outside directed moments of time and their content. We call it ' $\mathrm{e}$ ' in reference to the German word eigen and as employed in English words such as eigenvalue and eigenrhythm etc. It denotes something in its own right and hence an equivalent to an underlying source, given as a dynamic coherence provider, as discussed above.

In differentiating between coherent realised changes of states on the one hand and their hypostasised source on the other, we can map serial or parallel changes of states together with these sources. This allows us to map and symbolise a subjective dynamic in addition. More generally, we introduce a symbol linking verified past and present states and their anticipated future to a parallel and sequential continuity, although the temporal aspect of these states has a discrete, discontinuous quality. The corresponding symbol, then, helps us keep in mind the temporal uniqueness of the states investigated, without preventing to construe and to conceptualise these states in the form of a pseudo-continuity. Metaphorically speaking, we encapsulate time in a symbol. This way, we open the way to a formalised description of the basis of parallel and serial coherence, together with the realisations of this basis.

It is this basis of coherence that allows human beings to interact with their outside and inside world in a coherent and non-stochastic way. The fact that an underpinned coherence providing structure is the logical starting point of any encounter with the outside world causes an inevitable epistemic coherence bias. That is, as realised coherences, we tend to first interpret outside changes of states as being coherent also and underestimate the relative weight of stochastic changes. The suggestive, intriguing and seductive power of imaginary pseudo-coherences, as established in numerous holistic systems that hypostasise a primordial coherence of corresponding worlds, results from this bias.

\section{Conclusion}

In the three papers which follow we introduce the model step by step. To prove its applicability in both science and humanities, we use areas of study in both science and the humanities. Based on the combined objective and subjective perspectives, concepts such as salutogenesis and person-centered healthcare can be addressed in a formal and meaningful way. Our main concern was to formally map and to formally understand the interaction of psychic, also interpretative processes and bodily processes, as demonstrated for psychic stress and immediate, as well as delayed asthma reactions in children [3-5]. We welcome feedback on our approach.

\section{Acknowledgements and Conflicts of Interest}

Our four papers in this issue of EJPCH detail concepts and arguments presented at the First (2014) and Second (2015) Annual Conference of the European Society for Person Centered Healthcare in Madrid, Spain, as well as in poster presentations and workshop contributions at the 2013 and 2014 conferences of DEGAM (Deutsche Gesellschaft für Allgemeinmedizin und Familienmedizin), Munich and Hamburg, Germany. Our approach with reference to Helmuth Plessner's philosophy has been presented in a lecture at the IVth International Plessner Conference, Erasmus University, Rotterdam, The Netherlands, in 2009. The development of the model's mathematical basis has been presented at conferences of the European Academy of Allergy and Clinical Immunology EAACI in London and Geneva in 2010 and 2012. We declare no conflicts of interest. 


\section{References}

[1] Engel, G.L. (1977). The need for a new medical model: A challenge for biomedicine. Science 196, 129-136.

[2] Cloninger, C.R., Svrakic, D.M. \& Przybeck, T.R. (1993). A psychobiological model of temperament and character. Archives of General Psychiatry 50 (12) 975-990. [3] Sandberg, S., Paton, J., Ahola, S., McCann, D.C., McGuinness, D., Hillary, C.R. \& Oja, H. (2000). The role of acute and chronic stress in asthma attacks in children. Lancet 356, 982-987.

[4] Miall, D.S., Fröhlich, T. \& Henningsen, P. (1998). In the Convergence Zone: A Neuronal Model of Literary Response. European Journal of Neuroscience 10 (10) 305. [5] Froehlich, T., Bevier, F.F., Henningsen, P., Miall, D.S., Pompe, U. \& Sandberg, S. (2012). How words cause asthma. Allergy 67 (96) 315. 